\title{
Régimes thérapeutiques et dispositifs de preuve en oncologie : l'organisation des essais cliniques des groupes coopérateurs aux consortiums de recherche
}

Alberto Cambrosio*, Peter Keating ${ }^{\star \star}$ et Nicole Nelson ${ }^{\star \star \star}$

* Department of Social Studies of Medicine, McGill University, 3647 rue Peel, Montréal, QC, Canada H3A 1X1 ; ; alberto.cambrosio@mcgill.ca

** Département d'histoire, Université du Québec à Montréal, Case Postale 8888, succursale centre-ville, Montréal, QC, Canada H3C 3P8 ; keating.peter@uqam.ca

${ }^{\star \star \star}$ Department of the History of Science, University of Wisconsin-Madison, Bradley Memorial Building, 1225 Linden Drive, Madison, WI 53706-1528 USA ; ncnelson@wisc.edu

Résumé. À partir d'une analyse empirique du développement de l'oncologie médicale, et en particulier des essais cliniques, au cœur de ces pratiques, cet article examine la question du lien entre innovation organisationnelle et innovation épistémique. II explore comment les régimes thérapeutiques déployés par les cancérologues comptent parmi les actants principaux du style de pratique centré sur la performance d'essais cliniques, sans que l'on puisse dire si ces régimes relèvent du domaine de la connaissance ou de celui de l'organisation. L'article introduit la notion de regimen pour dépasser la distinction organisation-cognition. À près de 50 ans de distance, des solutions 
différentes, bien que reliées par une dépendance du sentier, on été mise en place en réponse à un même problème -l'accélération des échanges entre clinique et laboratoire. Nous sommes confrontés dans les deux cas à des réseaux sociotechniques très différents dont il faut prendre en compte la composition, ce que permet la notion de regimen. Notre étude est centrée sur l'oncologie, mais la notion de regimen pourrait offrir dans d'autres domaines une alternative à des notions comme celle de communautés épistémiques, et contribuer ainsi au dépassement de la distinction entre objets et pratiques.

À partir d'une analyse empirique du développement de l'oncologie médicale, et en particulier des essais cliniques qui sont au cœur d'une telle pratique, cet article examine la question du lien entre innovation organisationnelle et innovation épistémique. ${ }^{1}$ Plus précisément, nous nous intéressons à l'organisation en tant que composante des pratiques scientifiques : il s'agit, en d'autres termes, d'explorer le caractère mutuellement constitutif des modalités d'organisation et des pratiques scientifiques, en reprenant le postulat exprimé

\footnotetext{
${ }^{1}$ Cet article couvre de façon synthétique une période relativement longue, allant des années 1950 à aujourd'hui. Pour la période de l'après-guerre jusqu'au tournant du nouveau siècle, nous nous appuyons sur les données présentées dans notre livre (Keating et Cambrosio, 2012) auquel le lecteur pourra se référer pour des informations sur les sources d'information utilisées (archives, entrevues, analyse qualitative et informatisée de la littérature). Pour la période la plus récente nous utilisons les premiers résultats d'un projet en cours sur l'interface cancer et génomique en Europe et en Amérique du Nord, qui a recours à des approches ethnographiques (entrevues et observation) ainsi qu'à l'analyse de la littérature (articles scientifiques, newsletters électroniques). Pour plus de précisions voir Nelson et al. (2013).
} 
par Michel Callon (2012) : « organiser les pratiques scientifiques, c'est une pratique scientifique ». Faisant preuve d'un degré remarquable de réflexivité, des cancérologues ont d'ailleurs eux-mêmes posé la question de l'isomorphie entre évolution des structures organisationnelles et évolution des connaissances, et donc des liens mutuels entre innovation scientifique, clinique, et organisationnelle (Batist et Shinder, 2008). Analyser cette question nous oblige, cependant, à sortir des sentiers battus, en particulier en refusant de considérer les organisations et les connaissances comme deux entités distinctes et externes l'une à l'autre, qu'il s'agirait de mettre en relation. L’hypothèse selon laquelle il est difficile de séparer social et cognitif est maintenant bien établie grâce aux nombreuses études sur la science et la technologie, notamment celles se réclamant de la notion de co-production (Jasanoff, 2004). S'inscrivant dans ce cadre heuristique, ce texte explore comment les régimes thérapeutiques déployés par les cancérologues comptent parmi les actants principaux du style de pratique centré sur la performance d'essais cliniques, sans que l'on puisse dire si ces régimes relèvent du domaine de la connaissance (les essais visant à mieux comprendre la maladie et ses thérapies potentielles) ou de celui de l'organisation (les régimes, et les protocoles qui s'y rattachent, organisant les activités des oncologues). Le développement des essais cliniques en tant que dispositif central de l'oncologie passe par l'émergence d'organisations d'un type nouveau - les « groupes coopérateurs » - dont nous allons examiner les activités dans la première partie de l'article. L'oncologie médicale est cependant loin d'être un 
domaine statique ! Après avoir exploré la configuration simultanément épistémique et organisationnelle des premières décennies de la recherche clinique sur le cancer, nous rendrons compte de ses transformations récentes. Plutôt que d'opposer la modélisation statique d'une configuration sociotechnique à l'analyse de la dynamique du domaine, nous considérons que ces deux éléments participent d'un même cadre analytique lorsqu'on prend en considération l'alternance entre le cadrage fourni par les régimes et les débordements qui résultent de leur déploiement (Callon, 1998 ; 2002). Avant de mieux expliciter cet argument, il nous faut apporter quelques premières précisions empiriques.

\section{Les groupes coopérateurs comme organisations épistémiques}

Alors que les noms des centres de traitement du cancer, du moins des plus prestigieux d'entre eux (comme par exemple l'Institut Gustave-Roussy en France, ou le Memorial Sloan-Kettering aux Etats-Unis) sont bien connus du grand public, peu de lecteurs sont familiers avec des acronymes tels que ECOG, CALGB, SWOG, ou EORTC². Et pourtant, ces sigles un peu barbares renvoient à des institutions connues sous le nom générique de « groupes coopérateurs » (dans ce qui suit : les Groupes) qui, en parallèle avec les centres hospitaliers, ont contribué de façon décisive au cours des dernières décennies à

\footnotetext{
${ }^{2}$ Ces acronymes correspondent aux noms suivants : Eastern Cooperative Oncology Group, Cancer and Leukemia Group B, Southwest Oncology Group, et European Organisation for Research and Treatment of Cancer.
} 
la mise au point de nouveaux traitements contre les cancers et à leur traduction en routines cliniques. Le rôle des Groupes, en réalité, va bien au-delà de cette simple caractérisation. Ces organisations sont en grande partie responsables non seulement de l'introduction des chimiothérapies et des thérapies hormonales en tant que modalités de traitement du cancer, mais également de l'inscription au cœur de la cancérologie des essais cliniques en tant que forme spécifique de recherche médicale, et par là de l'émergence d'un nouveau style de pratique (Keating et Cambrosio, 2012). En effet, les essais cliniques en cancérologie, loin de se réduire à une simple routine méthodologique définie une fois pour toutes, sont des dispositifs très complexes, en constante évolution, dont la conception et la réalisation mobilisent des compétences multiples tout en générant de nouveaux types d'entités biocliniques, d'arguments et de justifications, de critères d'évaluation et de mises en équivalence, ainsi que de nouvelles formes d'objectivité et d'intersubjectivité. Bref, ils correspondent à une configuration sociotechnique (et donc organisationnelle, institutionnelle, matérielle, et conceptuelle) particulière, appelée à se transformer au fur et à mesure que ces mêmes dispositifs redéfinissent les routines cliniques. Une telle dynamique témoigne du caractère mutuellement constitutif de l'alternance routine/innovation.

Mais, en quoi consistent exactement les Groupes ? Même si le substantif « groupe » introduit d'emblée une dimension de continuité et stabilité qui va audelà des simples collaborations occasionnelles, l'épithète « coopérateurs » semble de prime abord renvoyer à une pratique très répandue dans le monde 
biomédical, à savoir des collaborations, souvent ad hoc et interinstitutionnelles, entre chercheurs et cliniciens. Mais les Groupes ont innové en renversant la séquence habituelle selon laquelle une collaboration découle de la nécessité de réunir plusieurs chercheurs pour mener à bien une expérience dont la conception précède la mise en place des liens de collaboration. Les pratiques coopératives institutionnalisées par les Groupes, et les infrastructures matérielles et conceptuelles qui les sous-tendent, constituent au contraire un point de départ pour la conception, avant même l'exécution, de projets de recherche clinique. Les Groupes s'apparentent, en d'autres termes, moins à une condition de possibilité qu'à une plateforme (Keating et Cambrosio, 2013) pour la conception et l'exécution d'essais cliniques.

Loin d'être des instances isolées ou indépendantes, les essais cliniques découlent et reposent sur les essais qui les ont précédés, et se projettent dans ceux à venir, s'insérant ainsi dans un programme de recherche ouvert dont les contours sont définis, justement, par la présence du dispositif des Groupes. On peut donc qualifier les Groupes d'organisations épistémiques car, comme nous venons de le suggérer, ils agissent en tant que plateformes pour un nouveau style de pratique. Dans la mesure où parmi les actants principaux du style de pratique centré sur la performance d'essais cliniques l'on retrouve les régimes thérapeutiques en tant qu'objets épistémiques et matériels, les essais ne sont pas un simple instrument au service d'un objectif de nature industrielle, à savoir tester la valeur thérapeutique de certaines molécules. Ils participent directement à la construction de l'objet de recherche lui-même (les cancers) et 
simultanément à la manière dont les tumeurs réagissent aux traitements, modifiant par là-même l'histoire naturelle de la maladie.

Quelques repères permettront au lecteur de cerner l'étendue du phénomène. À la suite d'un premier essai clinique randomisé effectué à l'Institut National du Cancer (NCl) des États-Unis en 1954, cette forme de recherche en cancérologie s'est développée très rapidement au cours des années 1960 et 1970, malgré I'hostilité initiale des secteurs les plus traditionnels du corps médical. Ces derniers ne voyaient pas d'un bon œil l'arrivée des chimiothérapies, qui concurrençaient les deux modalités de traitement alors dominantes (la chirurgie et la radiothérapie), et dont ils dénonçaient les effets secondaires ravageurs. Ils s'opposaient également au recours aux méthodes statistiques en milieu clinique, les comparant à un jeu de dés avec la survie des patients. Au cours des années 1980 et 1990 les essais cliniques, tout en se transformant grâce notamment à l'introduction de nouvelles méthodes statistiques et à l'ajout de nouvelles composantes, sont devenus une forme dominante de recherche clinique en oncologie, avec l'organisation d'essais s'étalant sur plusieurs années et réunissant des milliers de patients.

Responsables en grande partie de ces développements, les Groupes ont atteint au tournant du 21ème siècle une masse critique imposante, avec, en Amérique du Nord, plus de 10000 chercheurs-cliniciens repartis dans plus de 3000 institutions. En Europe, les 17 membres-fondateurs de ce qui devint par la suite I'EORTC (l'Organisation Européenne pour la Recherche et le Traitement du Cancer) posaient en 1962 les premiers jalons d'une organisation qui au début 
des années 2000 allait regrouper 2500 chercheurs distribués dans 300 institutions de 32 pays. Le succès de ces organisations d'un nouveau type s'est accompagné d'une complexification croissante de leurs activités. Des délais de plusieurs années s'étendent désormais entre la conception initiale d'un essai clinique et son exécution (Dilts et al., 2006). En conséquence, de nombreuses voix se sont récemment élevées, notamment aux Etats-Unis (IOM, 2010), pour réclamer une refonte du système des essais cliniques, accusé de ne plus correspondre aux exigences dictées par la recherche de pointe en cancérologie. Après plusieurs décennies de présence incontestée - bien que parfois critiquée - au centre de la recherche clinique en oncologie, les Groupes sont donc aujourd'hui confrontés à une remise en question de leur organisation comme de leurs activités. D'une part, on a assisté au cours des dernières années à une multiplication des consortiums réunissant des équipes ou des centres de recherche sur le cancer. Souvent consacrés à des projets particuliers et établis parfois sur une base ad hoc, mais parfois avec des visées plus stables et structurantes, ces consortiums ont en commun de promouvoir des modèles plus flexibles de coopération. Ils se veulent ainsi une solution de rechange aux modalités d'organisation incarnées par les Groupes. D'autre part, sous la houlette du $\mathrm{NCl}$ et dans un but de rationalisation les Groupes ont récemment procédé à des fusions qui ont réduit leur nombre de neuf à quatre, désormais intégrés à un réseau national d'essais cliniques établi sur le modèle du partenariat public-privé. Ce réseau - telle est la crainte exprimée par un porte- 
parole des Groupes - correspondrait plus à une infrastructure qu'à un dispositif de recherche (Schilsky, 2013).

Les Groupes ne sont cependant pas les victimes passives d'un projet de réorganisation et privatisation de la recherche. Comme nous le verrons, ils sont eux-mêmes en grande partie à l'origine des débordements qui ont conduit à la situation présente et, confrontés au défi des consortiums, ont rebondi en révisant leur structure et programme de recherche. Les débordements à l'origine de ces transformations peuvent être saisis par le terme de « recherche translationnelle », notion introduite au début des années 1990 pour désigner les travaux visant à relier recherche fondamentale et activités cliniques (Jones et al., 2011). De plus en plus présent à l'interface entre cancer et génomique, ce type de recherche se caractérise notamment par le fait d'intégrer au sein d'un même projet le spectre d'activités allant du laboratoire à la clinique.

Si le terme de recherche translationnelle remonte aux années 1990 et ne s'applique véritablement qu'à la période la plus récente, on retrouve une préoccupation semblable, à savoir accélérer la traduction clinique de percées précliniques, aussi bien aux Etats-Unis qu'en Europe au cours des années 1950 et 1960. L'émergence des Groupes dans l'après-guerre peut être analysée comme une réponse à cette préoccupation. Tout se passe donc comme si à plus de 50 ans de distance une même nécessité d'associer innovation organisationnelle et scientifique s'était fait jour, dans un premier temps en liaison avec l'émergence des chimiothérapies, et récemment avec la mise en 
place d'une oncologie post-génomique. Avant d'analyser plus en détail cette dynamique, il nous faut revenir à quelques considérations théoriques.

\section{Configurations et dynamique des transformations.}

II ne fait guère de doute que le travail de recherche - et la biomédecine ne fait pas exception - se caractérise de plus en plus par une dimension collective et distribuée. Les Groupes peuvent être analysés comme l'expression d'une telle tendance. Pour rendre compte de dynamiques proches de celles que nous venons de décrire, des chercheurs en sciences sociales ont proposé des notions faisant simultanément référence à des aspects sociaux et scientifiques. C'est le cas notamment des notions de « culture épistémique » (Knorr Cetina, 1999), de «communauté épistémique » (Haas, 1992), de «communautés de pratique » (Wenger, 1998), ou encore, de « communautés collaboratives » (Adler et al. 2008). Ces notions partagent une même parenté avec les notions jumelles de style et de collectif de pensée proposées en 1935 par Ludwik Fleck (2005), bien qu'à la différence de ces dernières elles tendent à inclure une dimension plus ouvertement politique. Cependant, alors que chez Fleck le style a préséance sur le collectif humain, qui n'est qu'un référent empirique permettant d'accéder au style, les versions récentes, à l'exception de Knorr Cetina, mettent surtout en avant la formation sociale, ce qui les rapproche d'une sociologie plus traditionnelle des professions (Abbott 1988), dont Eyal (2013) a montré les limites dès lors qu'il s'agit de rendre compte de pratiques mobilisant des 
expertises scientifiques et techniques. Ce qui nous intéresse, en revanche, ce sont moins les Groupes en tant que formations sociales, que le style de pratique qui les définit, et donc les essais cliniques sans lesquels les groupes n'auraient pas de raison d'être, ou, plus précisément, les régimes thérapeutiques en tant qu'actants centraux de ces essais. On peut ici tracer un parallèle entre les régimes et les mutations qui, comme l'a montré Bourret (2005) sont au centre des nouvelles pratiques d'oncogénétique, et ont abouti à la formation d'une « clinique des mutations » (Rabeharisoa et Bourret, 2009). Dans le cas présent, cependant, plutôt qu'à une clinique des régimes, nous montrons que cela a conduit à l'émergence des régimes en tant que nouveau type de clinique. ${ }^{3}$ Qu'entendons-nous par « régime »? Ce terme polysémique peut renvoyer, dans un sens étroit, à une combinaison de médicaments, incluant la façon de les administrer (régime thérapeutique) ou, dans un sens plus large, aux arrangements qui rendent possibles non seulement l'administration de ces régimes thérapeutiques, mais aussi leur conception et développement (cf. Moreira, 2012). Dans ce dernier cas, et bien qu'il s'agisse d'une notion empruntée aux praticiens, nous utiliserons le terme latin regimen pour signaler cet usage particulier. Comme nous allons l'expliciter, régimes et regimens sont étroitement liés, l'une ne saurait aller sans l'autre. Or, si le regimen joue le rôle central que nous lui attribuons, comment rendre compte du changement, en échappant à la solution traditionnelle qui consisterait soit à voir dans le changement de regimen la cause du changement de l'organisation des

\footnotetext{
${ }^{3}$ Nous remercions Pascale Bourret qui nous a suggéré ce renversement de perspective.
} 
Groupes, voire leur remplacement par d'autres assemblages organisationnels, soit dans ces changements organisationnels la cause des changements de regimen ? En définissant le regimen comme décrivant aussi bien la structuration des activités de recherche que l'objet de recherche, nous proposons une façon d'éviter cette impasse. Cette perspective, pour être efficace, doit s'étendre sur plusieurs décennies.

La constitution des Groupes dans l'immédiat après-guerre peut être analysée comme une réponse au problème de comment accélérer le passage du stade préclinique au stade clinique, et peut être vue, dans ce sens, comme un projet de recherche translationnelle avant la lettre. Par homologie, la reconfiguration présente du système des essais apparaît comme une réponse à un problème semblable mais redéfini par la présence d'une configuration sociotechnique fort différente, bien qu'en partie héritée des solutions mise en place par les Groupes dans l'après-guerre. Dans les deux cas, cependant, c'est moins le problème en tant que tel, voire les acteurs qui le formulent, qui sont à l'origine de cette continuité ; c'est la présence des régimes qui établit le lien conceptuel et matériel entre ces deux périodes. En d'autres termes, l'analyse ne saurait se centrer sur les formations sociales porteuses d'un tel questionnement : au contraire, elle doit faire place aux actants, dans notre cas les régimes, sur lesquels convergent les pratiques et à partir desquels elles se déploient. Dans cette perspective, nous disposons de deux approches différentes mais aisément conciliables. 
Daniel Navon (2013), en premier lieu, dans sa remarquable thèse sur les « désignations génomiques », propose la notion de « factualité réitérative », inspirée de l'approche en termes de « résolution réitérative de problèmes » (Haydu,1998). Variante historicisée de l'approche de la dépendance du sentier, celle-ci prévoit de contraster les solutions apportées à différentes périodes à des situations relevant d'un même type de problème. Mais Navon modifie de façon décisive cette approche en déplaçant l'analyse de la façon dont des acteurs sociaux construisent des problèmes dans des contextes historiques différents, à la façon dont des objets (dans son cas, les mutations) sont produits et mobilisés à différentes époques. Ceci implique une analyse des configurations sociotechniques qui agissent comme condition de possibilité pour la production des faits biomédicaux. Cette analyse est compatible avec la notion de réseau sociotechnique (Callon, 2002; Latour, 2007), dont elle se réclame d'ailleurs ouvertement. Ce qui nous amène, en second lieu, aux catégories analytiques de cadrage et débordement introduites par Callon (1998; 2002 ; voir aussi Callon et Rabeharisoa, 2008). Dans notre cas, nous verrons que le cadrage correspond, entre autres, à la mise en place d'un dispositif d'essais cliniques qui se décline par phases successives, de la Phase I destinée à vérifier la toxicité d'une substance, à la Phase III visant à déterminer l'efficacité d'un régime thérapeutique donné. Mais ce type de système bioclinique expérimental, qui redéfinit en même temps la maladie et ses traitements, donne lieu à des débordements qui brouillent cet ordonnancement et conduisent à la prolifération de nouvelles catégories (Phase 0, phases précoces, etc.) et au 
parasitage croissant de la distinction entre expérimentations et traitements. La suite de cet article est consacré à l'exploration de cette dynamique.

\section{L'émergence des groupes coopérateurs.}

D'aucuns ont analysé l'émergence des chimiothérapies anti-cancéreuses comme un exemple d'un présumé tournant industriel de la recherche biomédicale (Bud, 1978). La recherche américaine sur le cancer aurait notamment adopté les modèles des programmes centralisés de développement de la bombe atomique ou, plus près du monde médical, de la pénicilline et de lutte contre la malaria, déployés au cours de la deuxième guerre mondiale. Toutefois, une analyse centrée sur la structure et les activités des Groupes en tant que fer de lance du mouvement visant à introduire les chimiothérapies comme nouvelle modalité de traitement, permet de montrer que ceux-ci incarnent une forme distribuée de recherche, et que ce choix s'inscrivait en opposition directe et explicite avec une approche de type « industriel » et centralisé. Loin de correspondre à l'adoption d'un modèle préexistant qui aurait déjà fait ses preuves, la formule des Groupes a nécessité plusieurs années de bricolage, avant d'aboutir à une forme temporairement stable et codifiée.

Il existe en effet deux versions différentes de l'émergence des Groupes, une version « par le haut » et une version «par le bas », qui ne sont d'ailleurs pas incompatibles dans la mesure où elles capturent deux facettes d'un même 
processus. La première version insiste sur la mise en place d'un vaste programme de recherche clinique sur le campus des National Institutes of Health $(\mathrm{NIH})$ à Bethesda, incluant la création d'un centre hospitalier consacré aux thérapies expérimentales, où fut effectué en 1954 le premier essai clinique anti-cancer déjà mentionné. Un lobbying intense mené auprès du Sénat américain au début des années 1950 par une coalition d'activistes avait convaincu celui-ci de consacrer des montants accrus à la lutte contre le cancer dans l'espoir qu'une action concertée pourrait vaincre cette maladie (Patterson, 1987). Ces fonds furent utilisés, entre autres, pour créer un centre et un programme national de chimiothérapie anticancéreuse en liaison avec les NIH et le $\mathrm{NCl}$, dont la première tâche consista à passer au crible des millions de substances pour en évaluer les propriétés anticancéreuses, ainsi qu'à tester cliniquement les molécules ayant survécu aux épreuves de criblage. Mais les chercheurs et les cliniciens du NCl firent rapidement part de leur méfiance vis-àvis d'approches calquées sur le modèle des recherches de guerre, considérant qu'un tel modèle était inapproprié aussi bien d'un point de vue scientifique que psychologique, dans la mesure où toute recherche dirigée centralement manquerait nécessairement d'originalité et empiéterait sur l'autonomie des chercheurs. L'alternative, adoptée par le Sénat en 1954 sous la pression du NCl, consista à créer un réseau de centaines de chercheurs individuels (et non pas un réseau d'institutions), désigné par le terme de «système coopératif ». La structure exacte d'un tel système, et les modalités de contrôle qu'une instance 
centrale comme le $\mathrm{NCl}$ pouvait exercer sur celui-ci restaient cependant à définir, et elles ont effectivement évolué au cours des décennies successives.

La version «par le bas » insiste sur le fait que l'impulsion initiale pour la création des Groupes vint des chercheurs eux-mêmes, ces derniers ayant pu compter sur le soutien du NCl. Cette version, plus événementielle, fait référence au fait qu'à la suite du recrutement par une autre institution du chercheur-clinicien qui avait dirigé l'essai clinique du $\mathrm{NCl}$ en 1954, et afin de pouvoir poursuivre ce travail, il fut rapidement convenu d'officialiser la collaboration avec les collègues restés au $\mathrm{NCl}$, ce qui donna lieu à la création du premier Groupe, appelé «Leukemia Group B ». Établi par une poignée de chercheurs de deux institutions seulement, et donc comme une collaboration de nature conventionnelle, ce Groupe (par la suite rebaptisé « Cancer and Leukemia Group B » ou CALGB) allait compter en 1970 plus de cent chercheurs de 41 institutions, et conduire au type de renversement épistémique mentionné au début de ce texte. La création de ce premier Groupe donna lieu à une sorte de " dépendance du sentier ", dans la mesure où d'autres cliniciens, préoccupés par l'établissement de critères communs devant permettre d'évaluer la réponse aux traitements, créèrent peu de temps après le «Leukemia Group A ». Ces deux Groupes se spécialisant dans les hémopathies malignes, leurs collègues dans le domaine des tumeurs solides donnèrent rapidement naissance au «Eastern Solid Tumor Group » (par la suite rebaptisé « Eastern Cooperative Oncology Group» ou ECOG). En 1956 on comptait déjà huit Groupes, et au début des années 1960, 10 autres Groupes s'y étaient ajoutés. Plusieurs de ces 
Groupes allaient par la suite disparaître ou, dans certains cas, fusionner, au fur et à mesure que la configuration sociotechnique définissant l'organisation et les activités des Groupes se précisait et se stabilisait.

De l'autre côté de l'Atlantique, une version « par le bas » prédomine nécessairement, dans la mesure où la vingtaine de chercheurs de différents pays qui donnèrent naissance au début des années 1960 au « Groupe Européen de Chimiothérapie Anticancéreuse ", rebaptisé quelques années plus tard EORTC (« European Organisation for Research and Treatment of Cancer ») ne pouvaient compter sur la présence d'un équivalent européen du $\mathrm{NCl}$, sans parler de l'obstacle que représentait la multiplicité de juridictions nationales. II est vrai, cependant, que ce groupe de cliniciens-chercheurs qu'on pourrait qualifier, en empruntant le terme à Harry Marks (1999), de partisans d'une « réforme thérapeutique » doublée, dans ce cas, d'une vision pan-européenne, pouvait s'appuyer sur le précédent établi par leurs collègues américains, dont ils avaient pu découvrir les pratiques au cours de séjours plus ou moins étendus aux Etats-Unis. Le NCl interviendra d'ailleurs rapidement en soutenant financièrement leurs activités, palliant ainsi au manque de soutien des instances européennes.

Les Groupes, nous l'avons affirmé, constituent une réponse inédite au problème de comment structurer la recherche clinique, et ce aussi bien au niveau des modalités organisationnelles que du contenu des activités, ces deux éléments étant solidaires les uns des autres. Mais en quoi innovent-ils et, d'abord, sommes nous véritablement en présence de solutions innovantes ? On peut 
aborder cette dernière question en s'intéressant aux différentes façons dont les acteurs se sont interrogés sur leurs propres initiatives, qui laissent transparaître le caractère « bricolé » de leurs réponses, ou, si l'on préfère, une dynamique par essais et erreurs. Tout, ou presque, était à inventer, car les promoteurs des Groupes s'aventuraient dans une sorte de terra incognita. Ainsi, du côté européen, lors des rencontres à l'origine de l'EORTC, les membres fondateurs posèrent ouvertement la question de la nature de l'organisation à laquelle ils allaient donner naissance: s'agissait-il de créer une sorte de société savante, ou plutôt un groupe de travail, ou encore un réseau nécessairement restreint, du moins au début, dont l'objectif premier serait de s'engager dans des projets concrets de recherche ? Et, dans ce dernier cas, dans quel but et selon quelles modalités ?

Si la chimiothérapie s'imposait d'emblée comme l'horizon de recherche du nouveau collectif, il était loin d'être évident à l'époque quel cadrage, c'est-à-dire quel assemblage particulier de ressources, humaines et autres serait nécessaire pour s'engager dans cette voie, au-delà d'une profession de foi somme toute générique proclamant la nécessité de combiner recherche scientifique et clinique dans une sorte de recherche translationnelle avant la lettre. La nécessité de réunir une diversité d'expertises (des cliniciens, biologistes, chimistes, pharmacologues, endocrinologues, statisticiens, infirmières, etc.) fut signalée également par les pionniers du système américain. Le développement des chimiothérapies anticancéreuses, tout en nécessitant l'assemblage de différentes spécialités, allait aboutir à l'émergence, parfois contestée et souvent 
différée, d'une nouvelle spécialité, l'oncologie médicale, tout en provoquant l'éclosion de multiples fronts de recherche au sein des spécialités déjà établies. Ce processus est particulièrement évident dans le cas des statisticiens, avec l'émergence du domaine des bio-statistiques, tel que défini par la mise au point d'outils spécifiques à ce nouveau type de recherche clinique.

La conscience explicite de la nature hétérogène des ressources nécessaires à une telle entreprise, que l'on retrouve chez les pionniers de la chimiothérapie des deux côtés de l'Atlantique, se doublait de la conviction qu'il fallait, pour ce faire, produire de nouveaux dispositifs de recherche, même si un ensemble de facteurs institutionnels (en premier lieu l'existence du NCl) plaçait les débats américain et européen sur des plans très différents. Les cliniciens européens étaient, pour ainsi dire, condamnés à innover et bricoler, faute d'infrastructures déjà en place. Du côté des chercheurs américains, la présence d'une institution centrale forte coordonnant la recherche sur le cancer ne les empêcha pas d'explorer un certain nombre de possibilités. Ainsi, le dispositif des Groupes prévoyait au départ d'expérimenter avec différentes modalités, comme par exemple des Groupes « multi-protocoles », à l'existence en principe relativement stable dans le temps et ayant comme vocation de conduire plusieurs études de nature hétérogène, et des groupes « à un seul protocole ", plus volatiles et dont les activités étaient confinées à une seule étude. Un troisième modèle prévoyait l'établissement de trois groupes régionaux ayant recours à un protocole commun pour tester sur des patients d'une façon plus standardisée, et donc plus proche du criblage des médicaments que de la 
recherche clinique, les substances dont les propriétés anticancéreuses auraient auparavant été vérifiées sur les modèles animaux. À la fin, ce furent les Groupes «multi-protocoles » qui devinrent la norme.

La nature exploratoire des activités initiales des Groupes apparaît très clairement à la lecture, aujourd'hui parfois surprenante, des premiers articles publiés par leurs membres. Ainsi, les auteurs d'études parues en 1958 et en 1960 insistaient non pas tellement sur les résultats des essais qui, comme on peut s'en douter, étaient loin d'être spectaculaires, que sur les arrangements organisationnels qui les sous-tendaient. II s'agissait, en d'autres termes, de prouver la viabilité de cette nouvelle approche, en apportant la démonstration qu'il était tout à fait possible de recruter rapidement un nombre élevé de patients répartis dans plusieurs centres hospitaliers, de convaincre les cliniciens de ces centres de suivre un protocole commun et, surtout, de l'interpréter de façon semblable. II fallait aussi démontrer que le progrès des moyens de communication mettait désormais à la disposition des Groupes des outils efficaces (notamment les conférences téléphoniques) pour aboutir à la coordination entre les centres. En bref, il s'agissait de convaincre que l'organisation d'un essai clinique multi-centres, dont l'une des caractéristiques était, il faut le rappeler, de relier des individus agissant au sein de différentes institutions et non pas les institutions elles-mêmes, relevait du domaine du réalisable.

Une fois cette démonstration apportée, il restait à construire ce qu'on pourrait appeler, à la suite d'Amin et Cohendet (2004), l'architecture du système de 
production de ces nouvelles connaissances, grâce à une série d'investissements de forme (Thévenot, 1986). Un des plus connus est la distinction entre des essais de différentes « phases » (Phase I, II et III), qui dans sa version canonique, prévoit d'établir en Phase I le dosage et le degré de tolérance d'un traitement sur un petit nombre de patients, quelques dizaines au plus ; puis, en Phase II, des essais recrutant jusqu'à une centaine de patients fournissent des indications préliminaires sur son efficacité ; enfin, en Phase III des études comparatives et randomisées avec un nombre de patients pouvant atteindre plusieurs milliers, permet d'en attester ou infirmer les effets thérapeutiques. On pourrait de prime abord affirmer que cette séquence est un simple arrangement bureaucratique visant à assurer un emboîtement logique et ordonné entre les différentes étapes d'une recherche clinique, chacune dotée de ses propres ressources, outils et paramètres, mais également à doter ces pratiques d'une justification légale et éthique vis-à-vis, notamment, des instances de régulation, en minimisant les risques encourus par les patients. Ce serait sans compter sur le fait que le système des phases est moins le résultat d'une décision administrative, voire de pressions externes de la part des agences de régulation, que le résultat d'un bricolage qui se déroula sur plusieurs années en prenant des formes différentes des deux côtés de l'Atlantique avant de converger vers sa version actuelle, le souci principal étant d'assurer la robustesse des données produite par le dispositif. Moins le résultat d'une intervention administrative qu'un élément émergeant de la dynamique interne du nouveau style de pratique, le système des phases, tout comme d'autres 
composantes successives des essais cliniques, tels que les comités de suivi des données et de la sécurité (Keating et Cambrosio, 2009), témoignent de la construction progressive d'un dispositif complexe visant à produire un type particulier de faits portant sur un nouveau type d'objet bioclinique, le régime thérapeutique. Ce processus étendu dans le temps atteste du caractère consubstantiel des régimes thérapeutiques et des regimens, ces derniers définissant les plateformes d'où émergent les premiers.

II nous faut maintenant examiner un autre élément du dispositif, à savoir le protocole. II serait trompeur de se représenter ce dernier comme une simple séquence d'activités à suivre, d'abord parce que cela minimiserait la dimension proprement performative des protocoles - loin d'être le reflet d'activités préexistantes qu'ils se limiteraient à coordonner, ce derniers sont constitutifs des activités collectives qu'ils définissent - mais aussi parce que les protocoles ne se réduisent pas à une liste d'activités et, encore moins, à une traduction sous forme de schéma du plan de traitement. Ils correspondent à un processus qui, d'une part, comprend un ensemble d'éléments incluant les principes et les antécédents sur lequel l'essai clinique se base, la population visée et les interventions prévues, les paramètres encadrant le déroulement de l'essai, les critères permettant de mesurer telles ou telles variables, et ainsi de suite; et qui, d'autre part, renvoie à un ensemble d'institutions dont la création stabilise le dispositif des essais cliniques et assure ainsi l'exécution du protocole. Ces institutions incluent différents types de comités dont le but est de définir et évaluer les composantes du protocole, de l'approuver, de les rejeter ou d'exiger 
des modifications, d'en surveiller l'exécution (en le re-spécifiant de ce fait), activités qui à leur tour reposent sur le développement d'outils spécifiques d'audit, c'est-à-dire aussi bien des techniques statistiques que des banques de données et des formulaires standardisés agissant comme une chaîne de traduction entre les activités cliniques concrètes (par exemple, tel type de mesure, exécutée de telle façon) et les éléments abstraits que l'on retrouve dans le protocole. Ainsi, plutôt qu'une façon d'organiser des pratiques, le protocole est lui même une pratique aux multiples épaisseurs et prolongements. À ce niveau aussi tout était à inventer pour permettre et de relier un ensemble d'interventions, telles que prescrites par le protocole, à des méthodologies et à une organisation capables de les traduire en pratiques cliniques, et d'établir des équivalences entre les activités déployées dans les différents centres. Ainsi, comme le souligne un texte de la fin des années 1960 sur la méthodologie des essais cliniques (Zubrod et al., 1969), il s'agissait non seulement, comme nous l'avons déjà mentionné, de mieux cerner la « faisabilité et l'utilité » des collaborations en recherche clinique, mais, surtout, de « résoudre la question des critères communs et autres pièges sémantiques ». La nécessité d'établir des équivalences grâce à des critères communs, à laquelle fut consacrée une conférence à la fin des années 1950, fut en effet l'un des thèmes dominants des activités initiales des Groupes, dans la mesure où sans standards communs il ne pouvait y avoir de coopération. La recherche d'une solution à ce problème transita simultanément par la mise en œuvre de stratégies méthodologiques (définition de critères objectifs), de techniques décisionnelles (système de vote), 
d'approches organisationnelles (rencontres fréquentes), et d'outils techniques (utilisation du télex), tout en devant en même temps surmonter l'objection du manque potentiel de flexibilité d'un type de « recherche fondée sur des protocoles ». Car, si sans standards communs il ne pouvait y avoir de collaboration, trop de standardisation risquait d'aggraver le problème d'une désaffection possible (et partiellement constatée) parmi les membres des Groupes, confrontés à un type de recherche répétitive et sans intérêt pour le chercheur individuel, ce qui à son tour renvoyait au spectre de la recherche industrielle et donc aussi à une discussion plus générale sur la nature des essais cliniques : simples outils pour tester des substances ou véritables dispositifs de recherche?

Ces différents développements prirent un tournant organisationnel majeur avec la création, aussi bien aux Etats-Unis qu'en Europe (avec quelques variantes, même si l'approche européenne reprenait le modèle adopté aux États-Unis), d'unités centrales (des « centres de données ») pourvues de personnel en partie de type nouveau (notamment les gestionnaires de données). Ces unités centrales étaient chargées d'administrer les protocoles, c'est-à-dire en vérifier la faisabilité et le bien-fondé (en particulier statistique, afin de remédier au problème des essais abandonnés en cours de route ou dont les résultats étaient difficilement interprétables faute de pouvoir statistique), gérer les procédures de randomisation, recueillir et vérifier les données, mettre au point et d'effectuer les traitements statistiques, et générer des rapports. Les centres de données consacrèrent le rôle des statisticiens qui, de simples consultants, devinrent des 
collaborateurs à part entière, se voyant notamment attribuer par certains Groupes le privilège de figurer en deuxième place, après le responsable clinique, de la liste des auteurs des publications découlant d'un essai. En même temps, la création de ces unités impliquait nécessairement d'expérimenter avec des solutions inédites, non seulement parce que de telles unités (et une partie du personnel dont elles se composaient) n'existaient pas auparavant, mais aussi parce qu'elles mettaient au jour une tension toujours présente entre la nature distribuée des Groupes et la nécessité d'établir un certain degré de centralisation, ce qui ne fut pas sans créer quelques controverses, surtout en Europe. En d'autres termes, elles soulevaient de façon on ne peut plus directe la question de la coordination des activités et des mesures nécessaires pour assurer une telle coordination en présence d'une tension semblable à celle qui existe entre le modèle (centralisé et vertical) des organisations formelles et celui (distribué et horizontal) des méta-organisations (Ahrne \& Brunsson, 2006). Si le protocole définit les activités des Groupes, au point où l'on pourrait affirmer qu'il leur fournit simultanément une raison d'être et un dispositif permettant de l'actualiser, le protocole en soi, c'est-à-dire en tant qu'infrastructure générique, reste une coquille vide sans la présence d'un objet épistémique qui seul peut le faire accéder au statut de véritable plateforme de recherche. Contrairement à ce qu'une sorte d'empirisme naïf pourrait laisser croire, cet objet ne se réduit pas à une substance particulière, dont il s'agirait de tester les propriétés anticancéreuse, mais équivaut à ce que les membres des Groupes ont appelé des régimes thérapeutiques. Le champ sémantique de ce terme, qui à l'origine 
correspondait aux notions de contrôle et de gestion, renvoie à des règles de régulation de la vie par l'hygiène ou la diététique, aux traitements médicaux, mais aussi à une forme de gouvernance ou à un système politique. Ces multiples signifiés résument bien la portée de cette notion. Elle renvoie à la pratique qui structure les débats entre oncologistes et autour de laquelle se bâtissent les protocoles des essais cliniques. Elle renvoie simultanément à un outil de gouvernance de la vie (et de la mort : faire vivre/laisser mourir) dans un espace animé par de multiples actants (cliniciens, patients, molécules, techniques,...) et défini par la tension entre l'impératif d'innovation et l'impératif de soin (Baszanger, 2012). Ces deux impératifs se conjuguent dans l'espoir de guérison, au niveau du patient individuel comme à celui « mythologique » (Barthes, 1956), de la découverte du « remède contre le cancer ».

Tel qu'utilisé de façon routinière par les membres des Groupes, le terme régime renvoie dans la plupart des cas à une combinaison de substances, administrées selon des modalités particulières (à telle dose, de telle manière, à tel intervalle et pendant telle durée) et qui peut comprendre également d'autres interventions visant à réduire les effets secondaires (par exemple, des antiémétiques), ou encore, comme ce sera le cas dans les années plus récentes (voir la prochaine section), des mesures auxiliaires (par exemple des biomarqueurs) permettant de mieux cerner l'évolution des traitements. Qui plus est, les substances impliquées dans un tel régime ne sont pas des substances « naïves » ou, si l'on préfère, des entités purement biochimiques aux propriétés stables et intrinsèques, mais des substances ayant subi un processus d'enrichissement 
informationnel (Barry, 2005 ; Cambrosio et al., 2012) conséquent à leur passage par des épreuves multiples, comme les systèmes de criblage des médicaments dans des modèles animaux ou des essais cliniques antérieurs chez l'humain. Les régimes et les substances qu'ils déploient sous différentes formes et selon différentes combinatoires sont donc des objets relationnels, au sens où leurs propriétés sont définies et vont se modifier par rapport aux différents essais cliniques dont ils constituent la composante expérimentale. Ce qui nous renvoie au constat qu'un essai clinique n'existe pas de façon isolée, mais s'inscrit dans un ensemble d'essais visant à définir les propriétés de différents régimes (et de leurs variantes), ce qui ne saurait se faire sans redéfinir en même temps, sur la base de leur réaction aux régimes, les cancers contre lesquels ils sont dirigés. Un régime est donc un objet épistémique, et simultanément un objet clinique expérimental. Le qualificatif « expérimental » est important, car il renvoie , comme nous venons de le montrer, au fait que les activités des Groupes en déployant des régimes nichés au sein des protocoles s'attaquent aussi bien à l'exploration de traitements contre différents types de cancers qu'aux cancers eux-mêmes en tant qu'entités bio-pathologiques. Ainsi, si la réaction de certains types de tumeurs aux traitements peut conduire à la reclassification de ces tumeurs en différents sous-types, donc à de nouvelles entités nosologiques dont, à leur tour, on tiendra compte lors de la planification des essais successifs, il est également vrai que ce processus modifie simultanément la trajectoire biologique des tumeurs auxquels s'attaquent les oncologues : à l'histoire naturelle des tumeurs se substitue une histoire « traitée » des tumeurs. 
Il y a, en d'autres termes, coproduction des maladies et des essais cliniques, ce qui renforce encore la caractérisation des Groupes comme des institutions épistémiques.

\section{De nouveaux régimes ? Reconfigurer les essais cliniques à l'ère de la génomique}

Dans la première partie de ce texte nous avons examiné l'émergence du système des essais cliniques en parallèle avec la création et le développement des Groupes. Comme nous l'avons indiqué, avec la multiplication des régimes ce système a connu une croissance fulgurante au cours des années 1980 et 1990, période caractérisée par l'exécution de très nombreux essais cliniques de Phase III qui ont poussé à l'extrême l'analyse d'une combinatoire d'un nombre relativement restreint de molécules, avec des permutations parfois subtiles parmi les composantes de chacun de ces régimes. Nous n'allons pas nous attarder sur cette période, notre choix étant, pour cette deuxième partie de l'article, d'effectuer une avance rapide au début du $21^{\text {ème }}$ siècle.

En effet, si au cours de la deuxième moitié du $20^{\text {ème }}$ siècle le nombre de molécules anti-cancéreuses est demeuré faible (une trentaine à la fin des années 1980, une soixantaine dix années plus tard), à la fin de la première décennie du $21^{\text {ème }}$ siècle plus de 900 molécules expérimentales étaient en cours de développement et d'expérimentation. Une telle transition laisse entrevoir la présence d'un point tournant, caractérisé par un réajustement majeur du réseau 
sociotechnique des essais cliniques. Ce réajustement, qui s'applique aussi bien aux activités qu'à l'organisation des Groupes (les deux étant difficilement dissociables), et inclut également l'émergence de modes d'organisation alternatifs en dehors de ceux-ci, correspond à l'essor des thérapies dites ciblées, et, d'une façon plus générale, de ce qu'on appelle désormais la recherche translationnelle, souvent jumelée à des notions programmatiques comme celles de médecine personnalisée ou, encore, de médecine de précision. Le développement des thérapies ciblées redéfinit radicalement l'acteur-réseau au centre du système des essais cliniques, à savoir le régime thérapeutique.

Sans trop détailler, rappelons qu'au cours des 15-20 dernières années les connaissances sur la biologie des tumeurs et, en particulier, le développement d'outils permettant de l'analyser de façon fine (Rheinberger, 2000 ; 2009), ont fait des avancées très importantes, notamment en ce qui concerne l'identification des récepteurs à la surface des cellules cancéreuses et, d'une façon plus générale, des molécules impliquées dans les voies de signalement qui régissent l'émergence et le développement des tumeurs. Ceci a débouché sur la transformation tendancielle de maladies « communes », comme le cancer du sein, en un ensemble de maladies rares chacune définie par la présence ou l'absence de certaines entités moléculaires et réclamant donc, en principe, le recours à de régimes thérapeutiques spécifiques. Même si l'ancienne stratégie qui impliquait l'utilisation de régimes thérapeutiques visant à tuer de façon non sélective les cellules tumorales (affectant donc aussi les cellules normales, d'où 
les effets secondaires majeurs) demeure pour l'instant la composante principale des chimiothérapies anticancéreuses, elle a désormais fait place à la recherche de substances qui s'attaquent de façon spécifique aux molécules impliquées dans les voies de signalement tumorales (d'où le terme de « thérapies ciblées »). On voit clairement ici comme ce type de débordements qui redéfinissent l'objet des traitements (les tumeurs elles-mêmes) ébranle le cadrage établi par le système des phases. Ainsi, si les essais de Phase III constituaient l'activité principale des Groupes (et demeurent toujours un point de passage obligé pour l'octroi d'une AMM), l'attention des oncologistes s'est déplacée vers les études de Phase II ou, plus généralement, les essais de phase précoce, d'où émergent les nouvelles thérapies ciblées.

Cette dernière affirmation mérite d'être précisée. En introduisant la notion de regimen, nous avons évoqué le recours à des études auxiliaires, et notamment à I'utilisation de biomarqueurs. Or, si au cours des premières décennies d'activité des Groupes de telles études, conduites notamment par des pathologistes, s'inscrivaient au mieux dans un rapport de complémentarité, voire même d'extériorité par rapport à l'objectif principal des essais, au point qu'il serait hasardeux de les considérer comme une composante à part entière des regimens, avec les thérapies ciblées on assiste à une imbrication étroite des biomarqueurs et des nouvelles molécules. Dans la mesure où une molécule ciblée s'attaque, en principe, à une entité moléculaire spécifique, qui peut d'ailleurs se présenter sous sa forme originale ou mutée, il est impératif de vérifier si la tumeur du patient contient cette entité et sous quelle forme. Cette 
exigence est notamment à l'origine de l'émergence des tests appelés « diagnostics compagnons » qui constituent désormais, même au niveau des agences de régulation, une condition de possibilité pour la validation et I'utilisation des régimes ciblés. Par un effet de retour, cette saillance des biomarqueurs s'est étendue aux chimiothérapies traditionnelles, en donnant lieu à un nouveau type d'essais cliniques «fondés sur les biomarqueurs ». Et ce réassemblage s'est répercuté sur les autres éléments du réseau.

Nous avons mentionné dans la première partie de ce texte le développement du système des phases comme un pas important dans la création de l'architecture du nouveau mode de production des connaissances cliniques. Or, dès le milieu des années 1990, sous le coup de débordements que nous avons signalés, s'amorce une révision en profondeur de ce système, qui s'accélère au début du $21^{\text {ème }}$ siècle avec l'essor des thérapies ciblées. On en veut pour preuve, par exemple, la création d'une nouvelle phase, appelée Phase 0, consistant à administrer à des patients des micro-doses (non-thérapeutiques) d'un médicament ciblé expérimental dans le but de vérifier si cette molécule s'attache véritablement à la cible visée. Mais l'effet le plus frappant est la remise en question de la division classique en trois phases bien définies et mutuellement exclusives. Celle-ci a en effet fait place à un processus d'hybridation des phases, avec le recours à des essais de Phase I-II (visant simultanément à déterminer le dosage et la toxicité d'une thérapie ciblée et à recueillir d'entrée de jeu des indications sur son efficacité), ou encore la 
subdivision des essais de Phase II en essais de Phase lla et Ilb, les premiers s'apparentant à de études de Phase I-II et les deuxièmes à des Phases II-III. Cette reconfiguration du système des phases a donné lieu à de nouveaux investissements de forme, en Europe comme en Amérique du Nord, avec la création d'unités consacrées spécifiquement aux essais dits « de phase précoce ", dont l'objectif est de tester de façon transversale les nouvelles molécules expérimentales sur un ensemble de tumeurs, en privilégiant ainsi les anomalies moléculaires par rapport à la catégorisation traditionnelle des tumeurs par site histologique d'origine (sein, poumon, etc.).

D'autres types récents d'essais cliniques, dans le mesure où il visent surtout à étudier des biomarqueurs (en combinaison avec des régimes thérapeutiques) trouvent difficilement place dans les système des phases, au point qu'on peut les qualifier d'essais « sans phase » bien qu'ils continuent à être assujettis à ce système pour des fins de régulation. Par exemple, l'essai clinique français SAFIR 01 (André et al., 2012) mené dans 18 centres cliniques, se donnait trois objectifs : tester l'hypothèse que l'analyse à haut-débit du génome tumoral fournit plus d'informations que l'analyse des gènes pris individuellement ; examiner la possibilité d'établir un système national de plateformes génomiques devant permettre aux patients d'accéder aux traitements sur la base des anomalies moléculaires de leurs tumeurs ; et, enfin, détecter chez des patientes souffrant d'un cancer du sein la présence d'anomalies génomiques très rares dans ce type de cancer mais qu'on retrouve plus fréquemment chez d'autres 
types de cancers, en ouvrant ainsi la voie à l'utilisation hors AMM des régimes thérapeutiques ciblés approuvés pour ces derniers. Aussi bien par l'hétérogénéité des objectifs que par la présence d'actants comme les anomalies moléculaires et les techniques à haut-débit, on est très loin ici du design traditionnel des essais.

On assiste, en effet, à une éclosion de nouveaux types d'essais, dont les avantages et inconvénients font l'objet de discussions animées entre oncologistes. Mentionnons, notamment, l'essor remarquable au cours des dernières années des essais de type «néo-adjuvant ${ }^{4}$. Par contraste avec le cas beaucoup plus répandu des thérapies adjuvantes, administrées après l'exérèse de la tumeur afin d'éliminer toute cellule cancéreuse encore présente, l'approche néo-adjuvante consiste à administrer une chimiothérapie avant l'intervention chirurgicale afin de diminuer la taille de la tumeur et faciliter l'exérèse. Bien que relativement ancienne, elle est longtemps restée marginale, et ce n'est que récemment que le recours à ce type de design a connu un essor spectaculaire du au fait que, combinée avec des biopsies séquentielles, elle permet de tester (et éventuellement ajuster) directement sur les tumeurs in situ chez les patients les effets des molécules anticancéreuses, tout en suivant l'évolution des biomarqueurs sur les échantillons prélevés. D'un point de vue expérimental ceci offre une alternative à l'utilisation des modèles animaux, toujours imparfaits. Si cette approche a suscité des craintes parmi des

\footnotetext{
${ }^{4}$ Le mot-clé « neoadjuvant » renvoie à 67 publications recensées en 1998 dans PubMed et 1116 publications en 2011.
} 
bioéthiciens inquiets de l'utilisation répétée de biopsies (Peppercorn, 2013), des patients ont au contraire souligné le sens accru de contrôle sur leur propre traitement qu'il tiraient de ce suivi « en temps réel » de leur traitement. Une polémique assez virulente a ainsi opposé récemment des cliniciens critiquant un essai néo-adjuvant aux promoteurs de cet essai mais aussi aux patients qui les soutiennent (Schott et Hayes, 2012 ; Yee et al., 2012 ; Perlmutter et al., 2012). D’autres types de design ont été proposés, comme les essais dits de « fenêtre d'opportunité » (ou "essais fenêtre") qui consistent à greffer sur la phase initiale d'un essai de plus grande envergure une courte période d'une ou deux semaines pendant laquelle on administre, sans visée thérapeutique, un médicament expérimental. Bref, on ne peut qu'être frappé par les efforts déployés pour développer de nouvelles formes d'expérimentation.

Le système des Groupes a subi les contrecoups de ces multiples transformations. Un exemple est, à cet égard, très parlant. Aux Etats-Unis, à la suite des développements que nous venons de schématiser, des oncologistes ont organisé sous l'égide d'un des Groupes un essai clinique appelé I-SPY afin d'identifier des biomarqueurs pouvant prédire la réponse des patients aux traitements. Mais lorsqu'il fut décidé, à la suite des résultats de ce premier essais, d'en organiser un deuxième (I-SPY 2) visant à développer une approche de type néo-adjuvant pour évaluer rapidement des thérapies expérimentales en liaison avec des biomarqueurs, les responsables de ce nouvel essai qui s'inscrivait pourtant dans la droite ligne du premier, décidèrent de quitter le système des Groupes et de créer plutôt un consortium ad hoc, seul capable, à 
leur avis, de faire preuve de la flexibilité requise par le nouveau design. Ce dernier exige d'évaluer en temps réel les résultats des tests génomiques, et d'approuver et mettre en œuvre rapidement tout changement de protocole. II paraissait difficile d'incorporer de nouvelles technologies au cours de l'essai tout en gardant le cadrage traditionnel des Groupes, conçu pour des essais de Phase III de longue durée avec des protocoles fixes. Mais les Groupes ont rebondi. Ainsi, aux Etats-Unis, SWOG est responsable d'un essai « de nouvelle génération» (Master Protocol, Phase II-III) en partenariat public-privé, qui se propose de tester plusieurs substances simultanément en liaison avec des biomarqueurs. Plusieurs autres essais de ce type sont en préparation. En Europe, l'EORTC s'est faite le champion de la transition d'un modèle « empirique » des essais à un modèle « sur mesure » qui ne se limite pas à tester un régime thérapeutique contre une autre mais explore des questions plus proprement biologiques. Une des initiatives concrètes est l'essai MINDACT centré sur la validation d'une signature génomique (Kohli-Laven et al., 2011). Cet essai a demandé la mise en place d'une logistique très complexe pour permettre la circulation d'échantillons (de tissus, de sang, d'acides nucléiques) mais aussi de données informatisées entre différents centres européens, tout en respectant les différentes juridictions nationales et en essayant de minimiser le bouleversement pourtant nécessaire des routines cliniques des centres participants. Un des tout premiers résultats de cet essai a été la publication d'un article affirmant avoir fait la preuve qu'un essai clinique d'une telle complexité était faisable (Rutgers et al., 2011). Ceci n'est pas sans rappeler la publication 
des premiers articles des Groupes, évoquée au début de ce texte, dont l'objectif était d'apporter la preuve de la possibilité d'organiser des essais cliniques multicentres. Poursuivant sur cette lancée, l'EORTC a modifié sa structure traditionnelle constituée d'un ensemble de sous-groupes réunissant des oncologistes spécialisés dans un type donné de cancer, pour créer en 2005 un réseau regroupant un nombre restreint d'institutions traitant un grand nombre de malades et dotées de laboratoires sophistiqués, et devant justement permettre d'exécuter plus facilement de nouveaux types d'essais centrés sur des régimes de type ciblé.

Ces initiatives prennent place dans un paysage marqué par l'émergence de consortiums et réseaux biocliniques à l'extérieur du système des Groupes. C'est le cas, notamment, des réseaux ad hoc créés grâce à des financements ponctuels de la communauté européenne. Le réseau RATHER («Rational therapy for breast cancer») ${ }^{5}$, par exemple, implique des chercheurs et des cliniciens de six institutions publiques (universités et centres de recherche) et deux compagnies privées (dans les deux cas, des spin-offs de chercheurs de deux des institutions impliquées dans le réseau), et mise sur l'utilisation conjointe d'un ensemble de techniques à haut-débit (séquençage, expression génique, protéomique, etc.) afin d'identifier, dans le cas particulier de deux types de tumeurs difficilement traitables, des mutations pouvant servir de cible à des traitements. En partant de l'analyse d'échantillons de tumeurs annotés

\footnotetext{
${ }^{5}$ Des exemples d'autres consortiums du même type incluent RESPONSIFY (cancer du sein) et COLTHERES (cancer du colon).
} 
cliniquement et conservés dans des bio-banques (d'où l'importance d'inclure parmi les membres du réseau des chercheurs ayant accès à ce type de matériel) le projet s'étend jusqu'à la conception et l'exécution d'un essai clinique portant sur des substances dirigées contre les cibles identifiées par l'analyse (d'où l'importance d'inclure parmi les membres du réseau des cliniciens ayant accès à ces substances). Dans ces différents cas, on peut parler, par analogie avec les essais fondés sur les biomarqueurs, d'organisations fondées sur les biomarqueurs.

Ce panorama, nécessairement incomplet, nous donne néanmoins un aperçu des façons dont la transformation des regimens transforme l'organisation des pratiques. Les nouveaux regimens reliant étroitement biomarqueurs et substances ont engagé un processus de reconfiguration du système de la recherche clinique en oncologie, qui touche à ses différentes composantes. Mentionnons, d'abord, l'émergence (tout sauf purement conceptuelle) d'une distinction entre pronostic et prédiction, deux termes jusqu'à récemment considérés comme des synonymes. Le patient, sur la base d'une évaluation de paramètres cliniques et pathologiques (taille et diffusion de la tumeur, etc.) se voyait en effet assigner un pronostic plus ou moins favorable, ce qui allait ensuite déterminer la rigueur du traitement. Or, les biomarqueurs se déclinent désormais en deux catégories, des marqueurs de pronostic, devant permettre d'évaluer le cours de la maladie indépendamment du traitement, et des marqueurs prédictifs, devant permettre la réaction d'un patient (de sa tumeur) à un traitement donné (Kohli-Laven et al., 2011). Cette disjonction, souvent difficile 
d'application en pratique, ouvre la voie à plusieurs développements, notamment un éventuel déplacement du jugement clinique (Bourret et al., 2011), tout en générant de nouvelles contraintes et opportunités, car la validation de ces deux types de marqueurs exige le recours à des modalités différentes de preuve. La nécessité de valider les regimens associés à des biomarqueurs a provoqué une véritable éclosion d'essais au design innovateur. Nous avons évoqué les essais de type néo-adjuvant, mentionnons également le recours à des approches statistiques bayésiennes, jusqu'à récemment rejetées par la plupart des biostatisticiens en charge des essais, et qui demandent aussi l'utilisation de nouveaux critères permettant de juger rapidement des résultats de l'essai (comme la « réponse pathologique complète » plutôt que le taux de survie global). II serait faux de reléguer ces différents développements au « micro monde » de la clinique. A titre d'exemple, l'essai I-SPY 2 déjà évoqué est fortement impliqué et dans le domaine de la régulation (il a été un déclencheur de la publication par la Food and Drug Administration d'un projet de directive sur la « réponse pathologique complète »), et dans celui de la politique : l'essai a été nommément donné en exemple aux Etats-Unis lors de la présentation du nouveau Centre national pour l'avancement de la recherche translationnelle (Collins, 2011), mais aussi dans un document publié par la Maison Blanche et produit par le bureau des conseillers du président américain en matière de science et technologie (PCAST 2012). II devient difficile, ici, de distinguer où se termine la clinique et où commence le politique. 


\section{Conclusion}

Dans ce texte nous nous sommes intéressés au rapport entre innovation organisationnelle et innovation épistémique, en introduisant la notion de regimens (empruntée aux acteurs mais détournée à des fins analytiques) pour dépasser la distinction organisation-cognition. Le cas de l'oncologie médicale, examinée sur plusieurs décennies, se prête bien à une telle opération. En réponse à ce que l'on pourrait désigner comme un même problème - à savoir l'accélération des échanges entre clinique et laboratoire - nous pouvons observer, à près de 50 ans de distance, à la mise en place de solutions différentes, bien que reliées par une forte dépendance du sentier. Ces solutions, cependant, ne se laissent réduire ni à des stratégies d'acteurs ni à une sociologie politique des sciences qui fait l'économie des activités scientifiques elles-mêmes. En effet, nous sommes confrontés dans les deux cas à des réseaux sociotechniques très différents, dont il faut prendre en compte la composition, ce que permet justement la notion de regimen. Les changements qui ont affecté les Groupes, loin de se limiter à des modifications d'organigramme dans le seul but de rationaliser leurs opérations, concernent tout autant l'organisation des essais - les regimens - que les objets épistémiques vers lesquels ceux-ci convergent, à savoir les régimes thérapeutiques et les maladies contre lesquelles ces derniers sont dirigés et qu'ils contribuent à redéfinir. 
Les Groupes, dont nous avons esquissé le rôle et le développement dans la première partie de ce texte, ont contribué de façon décisive à la mise en place d'un cadrage qui, sous la pression des débordements décrits dans la deuxième partie du texte, a été remis en question par des assemblages qui font appel à des formes d'organisations plus flexibles s'inscrivant dans un paysage nosologique et pathologique profondément altéré. Tout se passe comme s'il y avait isomorphie croissante entre les nouveaux objets de la recherche en oncologie, à savoir les réseaux de signalement cellulaires, capables de se modifier lorsqu'une voie est bloquée par un médicament ciblé, et la recherche de modes d'organisation permettant de rajuster rapidement le tir dans un domaine caractérisé par la production de plus en plus rapide des données générées par les technologies post-génomiques.

Notre étude était limitée à l'oncologie, mais on peut se demander si la notion de regimen, au sens que nous lui donnons dans ce texte, pourrait s'étendre à d'autres domaines. Si tel était le cas, elle constituerait une alternative plus générale à des notions comme celle de communautés épistémiques, en contribuant au dépassement de la distinction entre objets et pratiques. Dans la mesure où le terme « régime » renvoie également à la gouvernance et à la politique (les congrès d'oncologie comprennent désormais des sessions sur « l'oncopolitique »), on peut également s'interroger sur les nouvelles conditions qui régissent les prises de décision technoscientifiques. Les limites de ce texte ne nous permettent pas de développer, mais nous soulignerons pour conclure que le regimen post-génomique implique de nouvelles relations entre médecins 
et patients, entre un nombre croissant de professionnels de la santé, entre organisations publiques et privées, entre agences de régulation et institutions de recherche. II renvoie dans ce sens à des pratiques qui sont à la fois scientifiques, morales, politiques et économiques, et ce de façon explicite et réflexive.

\section{Remerciements}

Les auteurs remercient les trois agences de recherche dont le soutien financier a rendu possible cet article : les Instituts de recherche en santé du Canada (MOP93553), le Fonds québécois de la recherche sur la société et la culture (SE124896), et le Conseil de recherches en sciences humaines du Canada (4102008-1833). Nous remercions également Michel Callon pour ses commentaires toujours stimulants et, en particulier, pour ses remarques concernant la notion de regimen et les liens entre organisation et pratiques scientifiques. Nous remercions enfin Pascale Bourret pour les très nombreuses discussions sur la génomique du cancer et plus particulièrement pour ses suggestions concernant ce papier. 


\section{RÉFÉRENCES BIBLIOGRAPHIQUES}

Abbott A., 1988, The system of professions. An essay on the division of expert labor, Chicago, University of Chicago Press.

Adler P. S., Seok-Woo K., Heckscher C., 2008, Professional work: the emergence of collaborative community, Organization Science, 19, 359-376.

Ahrne G., Brunsson N., 2006, Organizing the world, In : Djelic M. L., SahlinAndersson K., eds, Transnational governance: institutional dynamics of Regulation, Cambridge: Cambridge University Press, 74-94.

Amin A., Cohendet P., 2004, Architectures of knowledge: firms, capabilities and communities, Oxford, Oxford University Press.

André F., Nowak F., Arnedos M., Lacroix L., Viens P., Calvo F., 2012, Biomarker discovery, development, and implementation in France: a report from the French National Cancer Institute and cooperative groups, Clinical Cancer Research, 18, 155-1560.

Barry A., 2005, Pharmaceutical matters: the invention of informed material, Theory, Culture \& Society, 22, 51-69.

Baszanger I., 2012, One more chemo or one too many? Defining the limits of treatment and innovation in medical oncology, Social Science \& Medicine, $75,864-872$.

Barthes R., 1957, Le mythe, aujourd'hui, In : Barthes R., Mythologies, Paris, Seuil, 181-233. 
Batist G., Shinder G. A., 2008, The McGill University Department of Oncology: structure depicts the shape of evolving knowledge, Current Oncology, 15, 143-150.

Bazell R., 1998, Her-2: the making of Herceptin, a revolutionary treatment for breast cancer, New York: Random House.

Bourret P., 2005, BRCA patients and clinical collectives. New configurations of action in cancer genetics practices, Social Studies of Science, 35, 41-68.

Bourret P., Keating P., Cambrosio A., 2011, Regulating diagnosis in postgenomic medicine: re-aligning clinical judgment?, Social Science \& Medicine, 73, 816-824.

Bud R., 1978, Strategy in American cancer research after World War II: a case study, Social Studies of Science, 8, 425-459.

Callon M., 1998, An essay on framing and overflowing: economic externalities revisited by sociology, In : Callon M., ed., The laws of the market, Oxford, Blackwell, 244-269.

Callon M., 2002, From science as an economic activity to socioeconomics of scientific research: the dynamics of emergent and consolidated technoeconomic networks, In : Mirowski P., Sent, E. M., eds, Science bought and sold. Essays in the economics of science, Chicago: the University of Chicago Press, 277-317.

Callon M., 2012, Les sciences sociales face aux nouvelles pratiques de la recherche et de l'innovation, conférence donnée à Montréal, le 3 octobre. 
Callon M., Rabeharisoa V., 2008. The growing engagement of emergent concerned groups in political and economic life: lessons from the French Association of Neuromuscular Disease Patients, Science, Technology \& Human Values, 33, 230-261.

Cambrosio A., Keating P., Mogoutov A., 2012, What's in a pill? On the informational enrichment of anti-cancer drugs, In : Gaudillière J. P., Hess V., eds, Ways of regulating drugs in the $19^{\text {th }}$ and $20^{\text {th }}$ centuries, Houndmills, Palgrave Macmillan, 181-205.

Collins F. S., 2011, Reengineering translational science: the time is right, Science Translational Medicine, 3, 90cm17.

Dilts D. M., Sandler A. B., et al., 2006, Processes to activate Phase III clinical trials in a cooperative oncology group: the case of Cancer and Leukemia Group B, Journal of Clinical Oncology, 24, 4553-4557.

Eyal G., 2013, For a sociology of expertise: the social origins of the autism epidemic, American Journal of Sociology, 118, 863-907.

Fleck L., 2005 [1935], Genèse et développement d'un fait scientifique, Paris, Les Belles Lettres].

Haas P. M. 1992, Epistemic communities and international policy coordination, International Organization, 46, 1-35.

Haydu J., 1998, Making use of the past: time periods as cases to compare and as sequences of problem solving, American Journal of Sociology, 104, 339-371. 
IOM (Institute of Medicine), 2010, A national cancer clinical trials system for the 21st century: reinvigorating the NCl Cooperative Group Program, Washington, DC: The National Academies Press.

Jasanoff S., ed., 2004, States of knowledge. The co-production of science and social order. London: Routledge.

Jones D., Cambrosio A., Mogoutov A., 2011, Detection and characterization of translational research in cancer and cardiovascular medicine, Journal of Translational Medicine, 9:57.

Keating P., Cambrosio A., 2009, Who's minding the data? Data managers and data monitoring committees in clinical trials, Sociology of Health \& IIIness, $31,325-342$.

Keating P., Cambrosio A., 2012, Cancer on trial. Oncology as a new style of practice, Chicago: The University of Chicago Press.

Keating P., Cambrosio A., Nelson N., Mogoutov A., Cointet J. P., 2013, Therapy's shadow: a short history of the study of resistance to cancer chemotherapy, Frontiers in Pharmacology, 4:58.

Kohli-Laven N., Bourret P., Keating P., Cambrosio A., 2011, Cancer clinical trials in the era of genomic signatures: biomedical Innovation, clinical utility, and regulatory-scientific hybrids, Social Studies of Science, 41, 487-513.

Knorr Cetina K., 1999. Epistemic cultures: how the sciences make knowledge, Cambridge (MA), Harvard University Press.

Latour B., 2007, Changer de société, refaire de la sociologie, Paris: La Découverte. 
Marks H. M., 1999, La médecine des preuves. histoire et anthropologie des essais cliniques (1900-1990), Paris, Les Empêcheurs de penser en rond.

Moreira T., 2012, The transformation of contemporary health care. The market, the laboratory, and the forum, New York : Routledge.

Navon D., 2013, Genomic designation: new kinds of people at the intersection of genetics, medicine and social action, Thèse de doctorat, Columbia University.

Nelson N., Keating P., Cambrosio A., 2013, On being 'actionable': clinical sequencing and the emerging contours of a regime of genomic medicine in oncology, New Genetics \& Society, 32, 405-428.

Patterson J. T., 1987, The dread disease: cancer and modern American culture, Cambridge (MA), Harvard University Press.

PCAST, 2012, Report to the President on propelling innovation in drug discovery, development, and evaluation, Executive Office of the President of the United States (September).

Peppercorn J., 2013, Toward improved understanding of the ethical and clinical issues surrounding mandatory research biopsies, Journal of Clinical Oncology, 31, 1-2.

Perlmutter J., Axler S., et al., 2012, Advocates' perspective: neoadjuvant chemotherapy for breast cancer, Journal of Clinical Oncology, 30, 45864588. 
Rabeharisoa V., Bourret P., 2009. Staging and weighting evidence in biomedicine: comparing clinical practices in cancer genetics and psychiatric genetics, Social Studies of Science, 39, 691-715.

Rheinberger H. J., 2000, Beyond nature and culture: modes of reasoning in the age of molecular biology and medicine, In : Lock M., Young A., Cambrosio A., eds, Living and working with the new medical technologies. Intersections of inquiry, Cambridge, Cambridge University Press, 19-30.

Rheinberger H. J., 2009, Recent science and its exploration: the case of molecular biology, Studies in history and philosophy of biological and biomedical sciences, 40, 6-12.

Rutgers E., Piccart-Gebhart M. J., et al., 2011, The EORTC 10041/BIG 03-04 MINDACT Trial Is Feasible: Results of the Pilot Phase, European Journal of Cancer, 47, 2742-2749.

Schilsky R. L., 2013, Publicly funded clinical trials and the future of cancer care, The Oncologist, 18, 232-238.

Schott A. F., Hayes D. F., 2012, Defining the benefits of neoadjuvant chemotherapy for breast cancer, Journal of Clinical Oncology, 30, 17471749.

Thévenot L., 1986, Les investissements de forme, In : Thévenot L., ed., Conventions économiques, Paris, Presses Universitaires de France (Cahiers du Centre d'Étude de l'Emploi), 21-71.

Wenger E., 1998, Communities of practice: learning, meaning, and identity, Cambridge (MA), Harvard University Press. 
Yee D., Haddad T., et al., 2012, Adaptive trials in the neoadjuvant setting: a model to safely tailor care while accelerating drug development, Journal of Clinical Oncology, 30, 4584-4586.

Zubrod C. G., Schneiderman M., et al., 1969, Appraisal of methods for the study of chemotherapy of cancer in man: Comparative therapeutic trial of nitrogen mustard and triethylene thiophosphoramide, Journal of Chronic Disease, 11, 7-33.

\section{Abstract}

\section{Therapeutic regimens and proofs in oncology: the organization of clinical}

\section{trials from cooperative groups to research consortia}

Grounded in an empirical analysis of the development of medical oncology from the 1960s-present, this paper explores the mutually constitutive relation between epistemic and organizational innovation. In particular, it examines how, confronted with a 'similar' issue - the promotion of stronger interactions between pre-clinical and clinical work-1960 oncologists and their turn-of-thecentury colleagues developed different solutions, in spite of powerful pathdependency effects. These different outcomes cannot be reduced to the actors' strategies or to a political sociology of science that neglects the content of bioclinical practices. They correspond to two distinctive socio-technical networks that can be better understood by focusing on oncology's regimens, and the sequence of framings and overflows they engender. Social scientists continue to debate whether organizational innovation leads to cognitive innovation or vice 
versa, and are still caught in the opposition between configurational (static) and dynamic analysis. Regimens provide a heuristic alternative to these dichotomies. It remains to be seen whether this approach can be applied to domains other than oncology, thus bypassing the opposition between objects (entities) and practices.

\section{Resumen}

\section{Regímenes terapéuticos y dispositivos de prueba en oncología: la}

\section{organización de los ensayos clínicos de los grupos cooperativos a los consorcios de investigación}

A partir de un análisis empírico acerca de la evolución de la oncología médica, y particularmente de los ensayos clínicos que se encuentran en el corazón de tal práctica, este artículo estudia la cuestión de la relación entre la innovación organizativa y la innovación epistémica. En particular, este trabajo explora la manera en qué los regímenes terapéuticos realizados por los oncólogos cuentan entre sí a los principales actantes de un estilo de práctica centrado en el rendimiento del ensayo clínico, sin que se pueda decir si estos regímenes se encuentran dentro del área del conocimiento o de la organización. Así, el artículo introduce el concepto de regimen (noción tomada de los actores pero desviada para fines analíticos) para superar la distinción organizacióncognición. Con casi 50 años de diferencia, diferentes soluciones, aunque unidas entre sí por una marcada dependencia de la trayectoria, han sido introducidas en respuesta al mismo problema - la aceleración de los intercambios entre clínica y laboratorio. Nos enfrentamos en ambos casos con redes socio- 
técnicas muy diferentes, de las cuales hay que tener en cuenta la composición, lo cual permite justamente la noción de regimen. Nuestro estudio se centra en la oncología, pero podríamos extender el concepto de regimen a otras áreas, ofreciendo una alternativa a nociones tales como la de comunidades epistémicas, contribuyendo así a la superación de la distinción entre los objetos y las prácticas. 